\title{
THERAPEUTIC EVOLUTION IN ADULT-ONSET STILL DISEASE AT A REFERENCED CENTER OF RHEUMATOLOGY IN SÃO PAULO
}

Natália Carneiro Dos Santos (HSPE-IAMSPE, São Paulo, SP, Brasil), Ana Paula Dauricio de mattos (HSPE-IAMSPE, São Paulo, SP, Brasil), Ana Clara Ribeiro (HSPE-IAMSPE, São Paulo, SP, Brasil), Marcelo Nora Resende (HSPE-IAMSPE, São Paulo, SP, Brasil), Tamires Guimarães Costa (HSPE-IAMSPE, São Paulo, SP, Brasil), Sandra Echazu Roman (HSPE-IAMSPE, São Paulo, SP, Brasil), Roberta De Almeida Pernambuco (HSPE-IAMSPE, São Paulo, SP, Brasil), Rina Dalva Neubarth Giorgi (HSPE-IAMSPE, São Paulo , SP, Brasil)

\section{BACKGROUND}

Adult-onset Still Disease (AOSD) is a chronic inflammatory disease of unknown etiology that commonly affects young adults. Treatment of AOSD patients includes non-steroidal anti-inflammatory drugs, corticosteroids and disease-modifying antirheumatic drugs (Methotrexate, Leflunomide, Azathioprine, Cyclosporine, Cyclophosphamide). And, recently, biological drugs such as anti-IL1, anti-TNF and anti-IL6 are being included as a treatment option.

Anti-TNFs, such as infliximab and ethanecept, appears as an option for refractory cases to the use of glucocorticoids associated with an antirheumatic drug (mainly with methotrexate). And, currently, the use of anti-interleukins (mainly IL6) has been highlighted in cases of difficult activity control, in monotherapy or in association with classical drugs.

After a literature review of patients with refractory AOSD and use of anti-IL6, it was observed that this drug represents a great advance in the management of these patients.

\section{MATERIALS AND METHODS}

Observational, cross-sectional, retrospective study. A review of the medical charts of all patients diagnosed with AOSD from 2008 to 2018, followed-up at a referenced center of rheumatology in São Paulo. Adult patients who fit the diagnosis according to the criteria of Yamagushi et al. ( were included Patients who lost follow-up or had the diagnosis changed during the follow-up were excluded.

\section{RESULTS}

The total sample was 16 patients, $62.5 \%$ female. The mean age at the onset-disease was 53.7 years old (ranging from 39 to 73 years old). The most frequent criteria at the diagnosis were fever $(100 \%)$, leukocytosis with neutrophilia (87.5\%) and polyarthritis (81.2\%). All patients underwent treatment with methylprednisolone pulse therapy followed by maintenance of oral corticosteroid. $56.2 \%$ of the studied patients had the association of methotrexate to the therapy, while $25 \%(n=4)$ had started treatment with a combination of methotrexate + tocilizumab + oral corticoid. A total of $62.5 \%$ of patients $(n=10)$ presented remission of disease with the initial treatment, while $18.7 \%(n=3)$ failed. Then, it was chosen to associate the inhibitor of IL- 6 to these patients, who presented a good clinical-laboratory response, with $100 \%$ of remission of the disease.

\section{CONCLUSION}

As previously reported in the literature, some patients have a difficult-to-control disease with the use of conventional immunomodulators alone, therefore, tocilizumab emerged as a very satisfactory biological treatment option, as observed in the study, with index of success of $100 \%$ among patients who started using the anti-interleukin 6 after failure with methotrexate and oral corticosteroid. 